\title{
Stratification of Highest-Risk Patients with Chronic Skin Ulcers in a Stanford Retrospective Cohort Includes Diabetes, Need for Systemic Antibiotics, and Albumin Levels
}

\author{
Omar Amir, ${ }^{1}$ Andy Liu, ${ }^{2}$ and Anne Lynn S. Chang ${ }^{1}$ \\ ${ }^{1}$ Department of Dermatology, Stanford University School of Medicine, Pavilion C, 2nd floor, 450 Broadway Street, MC 5334, \\ Redwood City, CA 94063, USA \\ ${ }^{2}$ Department of Medicine, Albert Einstein College of Medicine, 1925 Eastchester Road 15A, Bronx, New York City, NY 10461, USA
}

Correspondence should be addressed to Anne Lynn S. Chang, alschang@stanford.edu

Received 31 July 2012; Accepted 10 September 2012

Academic Editor: Marco A. C. Frade

Copyright ( $) 2012$ Omar Amir et al. This is an open access article distributed under the Creative Commons Attribution License, which permits unrestricted use, distribution, and reproduction in any medium, provided the original work is properly cited.

\begin{abstract}
Chronic nonsurgical skin wounds such as venous stasis and diabetic ulcers have been associated with a number of comorbid conditions; however, the strength of these associations has not been compared. We utilized the Stanford Translational Research Integrated Database Environment (STRIDE) system to identify a cohort of 637 patients with chronic skin ulcers. Preliminary analysis $(n=300)$ showed that $49.7 \%$ of the patients had a poor prognosis such as amputation or a nonhealing ulcer for at least a year. Factors significantly associated $(P<0.05)$ with these outcomes included diabetes mellitus, chronic kidney disease, peripheral neuropathy, peripheral arterial disease, and need for systemic antibiotics. Patients with poor outcomes also tended to have lower hemoglobin levels $(P=0.01)$, higher WBC levels $(P<0.01)$, and lower albumin levels $(P<0.01)$. On multivariate analysis, however, only diabetes mellitus (OR 5.87, 1.36-25.3), need for systemic antibiotics (OR 3.88, 1.06-14.2), and albumin levels (0.20 per unit, 0.07-0.60) remained significant independent predictors of poor wound-healing outcomes. These data identify patients at the highest risk for poor wound-healing and who may benefit the most from more aggressive wound care and treatment.
\end{abstract}

\section{Introduction}

Chronic wounds cause a significant morbidity and financial expense in the United States, affecting 6.5 million patients with estimated treatment costs of $\$ 25$ billion per year $[1,2]$. Venous leg ulcers, the most common type of chronic skin wound, alone affect more than 1 million US citizens per year with an associated annual cost of $\$ 2.5$ billion $[3,4]$. Of these patients, only $50 \%$ effectively heal, affecting both quality of life and requiring long-term care. Moreover, in the diabetic population, numbering approximately 17 million patients in the United States, nonhealing foot ulcers can become life threatening if infected and confer a 15\% increased risk of amputation compared to the general population [5-8].

A number of factors have been documented in the medical literature which predispose patients to poor wound healing. These include underlying diseases such as diabetes mellitus, venous insufficiency, peripheral arterial disease, tobacco smoking, low serum albumin, and inflammatory conditions (such as pyoderma gangrenosum) among others [8-15]. According to a recent report, chronic kidney disease (CKD), hypertension, and myocardial ischemia may also be associated with increased risk of developing foot ulcers including severe ulcers that necessitate amputation $[16,17]$. Additionally, there are reports of higher rates of malnutrition and deficiencies of vitamins and minerals such as zinc in patients with chronic venous leg ulcers compared to the general population [18-20].

Although risk factors for the development of skin ulcers have been identified, clinical indicators of poor wound healing are less well studied. There are no large, well-controlled studies on independent impact of multiple risk factors including demographic, clinical, and laboratory markers to prognosticate outcome. In this study, we seek to stratify the level of risk which comorbidities and laborators values may confer on poor wound healing. If these markers can be 
identified, at-risk patients can be better identified and treated in a way that more aggressively addresses their comorbid medical condition, thus increasing the likelihood for effective wound healing.

\section{Methods}

2.1. Cohort Selection. Following Stanford Institutional Review Board approval, we employed a retrospective cohort study design using the Stanford Translational Research Integrated Database Environment (STRIDE) system. STRIDE includes data from Stanford University Hospital and Clinics and the Lucile Packard Children's Hospital (LPCH). It encompasses 13 years of clinical documents with information on medical diagnoses (including classification by ICD-9 codes), laboratory values, medications, radiology reports, pathology reports, and free text of progress notes, consultations, and discharge summaries. Previously, STRIDE has been successfully utilized to construct cohorts based on ICD-9 codes and laboratory values. In particular, the Stanford Dermatology Department used STRIDE to identify co-morbid medical conditions associated with transaminitis in psoriasis patients taking methotrexate.

The STRIDE Cohort Discovery Tool was used to select a cohort of patients with chronic skin ulcers. The following ICD-9 codes were used to select the cohort: 707.10-19, 785.4, $454.0,454.2$, and 440.23. These pertain to "unspecified ulcer of lower limb," "ulcer of thigh," "ulcer of calf, "ulcer of ankle," "ulcer of heel and midfoot," "ulcer of other part of foot," and "ulcer of other part of lower limb", respectively. The following text restrictions were used to further ensure that all patients included in the cohort had documentation of skin ulcer in physician-authored clinical notes: "ulcer," "wound," "erosion," "breakdown," and "gangrene." Patients aged 18 or older who had incident cases of skin ulcers between January 1, 2002 and January 1, 2005 were included. In the case of multiple ICD-9 codes meeting inclusion criteria only data on earliest ulcer within our date restrictions was used for the analysis.

2.2. Clinical Data Abstraction. Clinical data on exclusion criteria, outcomes, predictors, and covariates was extracted from STRIDE using its Data Review Tool. The Data Review Tool allows for optimized electronic chart review using string searches and filters based on prespecified criteria.

2.3. Exclusion Criteria. The following exclusion criteria were used based on manual review of clinical charts using STRIDE's data review tool: (1) no confirmation of ICD9 code diagnosis with clinical documentation, (2) pressure ulcer, (3) oral or mucosal ulcer, (4) primary dermatitis rather than skin wound, (5) ulcer is a primary skin infection or cellulitis, (6) ulcer is actually a primary surgical wound, (7) thrombophlebitis without ulceration, (8) ulcer is actually not an ulcer but a deep vein thrombosis without ulceration, (9) ulcer is actually a fistula, and (10) malignancy within wound.
2.4. Outcome Assessment. The patients were followed for 1 year out from the date of diagnosis as documented in physician-authored clinical notes. They were assessed on chart review for wound outcomes. A healing wound or good wound-healing outcome was defined as a wound that was documented by a physician to have healed within 1 year of followup. A nonhealing-wound or poor wound-healing outcome was characterized as either (a) a wound that had not healed by 1 year of followup, (b) a wound that required amputation, or (c) a wound that required flap reconstruction over the followup time. Patients whose wound status was unknown after 1 year of followup were characterized as lost to followup. These were subdivided into those who died, those who returned to Stanford subsequently but lacked an update on wound status on clinical chart review, or those who were never again seen at SUH. All patients who were lost to followup were excluded from subsequent data analyses.

2.5. Data Collection. Using the STRIDE data review tool, we manually collected data on demographic and clinical variables including laboratory values. In the case of laboratory values with multiple entries over time, only the lab value which was closest to the date of diagnosis of the ulcer within a 3-month time window of date of diagnosis was used. We additionally collected data on age at ulcer diagnosis, sex, race, current smoking, maximum dimension of wound at diagnosis, and preexisting duration of wound at diagnosis. We also collected data on treatments administered for the wound at Stanford University Hospital and Clinics.

2.6. Data Analysis. We conducted bivariate analyses comparing mean (parametric variables) or median (nonparametric variables) values of continuous predictors among healing and non-healing wounds, using ANOVA or KruskalWallis tests as appropriate. The association of dichotomous variables with the outcome was assessed using the $\chi^{2}$ test or Fisher's exact test for cell counts $<5$. Finally multiple logistic regression models were used to assess the independent impact of predictors. Only those predictors that were significantly associated with the outcome on univariate analyses were selected for the multivariate regression models. A $P$ value of 0.05 was used for all analyses.

\section{Results and Discussion}

As illustrated in Figure 1, using the STRIDE cohort discovery tool, a total of 637 patients with skin ulcers who were aged 18 or older were found to have been seen at Stanford University Hospital and Clinics between January 1, 2002 and January 1, 2005. Data for 300 of these patients was manually reviewed in the STRIDE data review tool in preliminary analysis. Out of these, $89(29.7 \%)$ were excluded based on our prespecified exclusion criteria leaving 211 (70.3\%) patients. From these, 76 patients $(25.3 \%)$ were lost to followup before 1 year. The remaining $135(45 \%)$ had complete data on wound outcomes over 1 year and were included in the final data analysis. Out of these 135 patients, $68(50.3 \%)$ patients had wounds that healed within 1 year and 67 patients $(49.7 \%)$ had wounds 


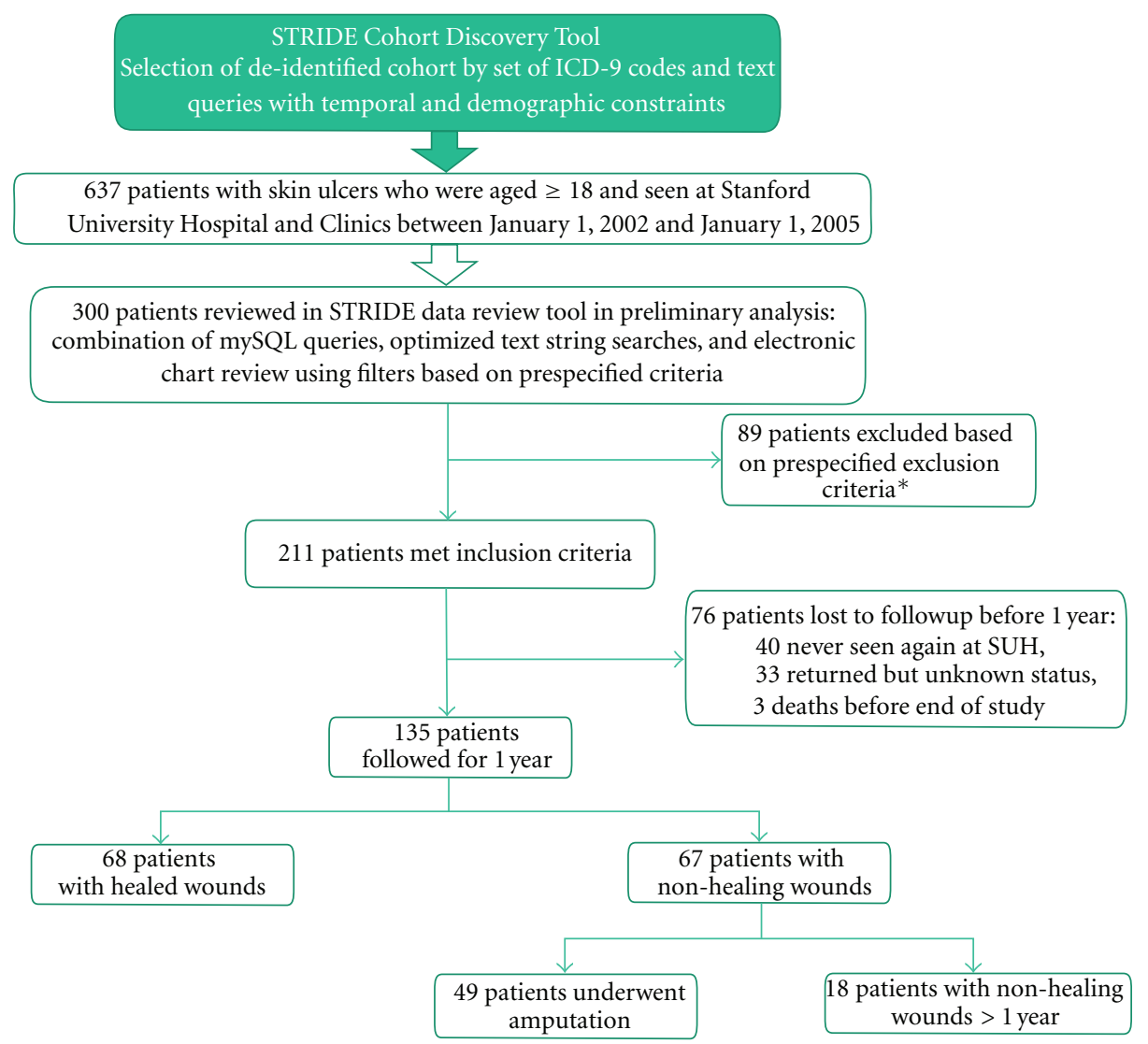

FIGURE 1: Cohort selection and refinement algorithm and results.

TABLE 1: Demographics and characteristics of patients with healing and non-healing wounds ${ }^{1}$.

\begin{tabular}{|c|c|c|c|}
\hline & Healing wound & Nonhealing wound & $P$ value \\
\hline Total number of patients & 68 & 67 & \\
\hline $\operatorname{Age}^{2}$ (years) & $63.7 \pm 15.6$ & $67.5 \pm 14.8$ & 0.21 \\
\hline Gender (\% male) & $32 / 68(47.7 \%)$ & $34 / 67(50.1 \%)$ & 0.92 \\
\hline Race (\% white) & $49 / 68(73.1 \%)$ & $47 / 67(69.1 \%)$ & 0.61 \\
\hline Smoking ${ }^{3}$ (\% current smokers) & $8 / 66(12.1 \%)$ & $7 / 57(12.2 \%)$ & 0.83 \\
\hline Size of wound ${ }^{4,5}(\mathrm{~cm})$ & $1.5(1.0-4.0)$ & $1.7(1.0-5.5)$ & 0.78 \\
\hline Preexisting duration ${ }^{6,7}$ of wound (months) & $1.0(0-2)$ & $1.0(0-3)$ & 0.11 \\
\hline \multicolumn{4}{|l|}{ Location of wound } \\
\hline Lower extremity (\%) & $63 / 68(94.0 \%)$ & $66 / 67(98.5 \%)$ & \\
\hline Upper extremity (\%) & $3 / 68(4.4 \%)$ & $1 / 67(1.4 \%)$ & 0.52 \\
\hline Other location (\%) & $1 / 68(1.5 \%)$ & $0 / 67(0 \%)$ & \\
\hline
\end{tabular}

${ }^{1}$ Healing wounds were those that healed within 1 year of followup. Non-healing wounds were defined as wounds that required amputation or did not heal after 1 year of followup. ${ }^{2}$ Data expressed as mean \pm standard deviation. ${ }^{3}$ Data based on smaller subsample of $n=128$ due to missing data. ${ }^{4}$ Data based on smaller subsample of $n=72$ due to missing data. ${ }^{5}$ Size expressed as median \pm interquartile range of maximum dimension of wound at presentation. ${ }^{6}$ Data based on smaller subsample of $n=93$ due to missing data. ${ }^{7}$ Wound duration expressed as median \pm interquartile range.

that did not heal after 1 year. Among these 67 patients with non-healing wounds, 49 patients underwent amputation and 18 patients had wounds that were documented to be nonhealing after 1 year. No patients underwent surgical flap reconstruction for the wound. For healing ulcers, median time to healing was 2 months with an interquartile range of 1-5 months (data not shown).
Table 1 shows the demographic and clinical characteristics of patients with healing and nonhealing wounds. On bivariate analyses, the distribution of demographic factors like age or gender did not vary significantly with the outcome. Current smoking did not significantly predict a poor wound-healing outcome. In addition, clinical characteristics of the wound such as preexisting wound duration and size 
TABLE 2: Clinical risk factors ${ }^{1,2}$ associated with non-healing wounds ${ }^{3}$.

\begin{tabular}{lccc}
\hline & Healing wound $(n=68)$ & Nonhealing wound $(n=67)$ & $P$ value \\
\hline Diabetes mellitus (\%) & $28 / 68(41.8 \%)$ & $55 / 67(82.1 \%)$ & $<1 / 67(76.1 \%)$ \\
Peripheral neuropathy (\%) & $28 / 66(42.4 \%)$ & $36 / 67(53.7 \%)$ & $<0.001^{*}$ \\
Renal insufficiency (\%) & $21 / 67(31.3 \%)$ & $48 / 65(73.8 \%)$ & $0.008^{*}$ \\
Peripheral arterial disease (\%) & $35 / 67(52.2 \%)$ & $21 / 63(33.3 \%)$ & $0.02^{*}$ \\
Venous stasis (\%) & $32 / 65(49.2 \%)$ & $24 / 61(39.3 \%)$ & 0.067 \\
Congestive heart failure (\%) & $23 / 65(35.3 \%)$ & $13 / 68(19.1 \%)$ & 0.65 \\
Immunosuppression $^{3}(\%)$ & $15 / 67(22.4 \%)$ & $3 / 67(4.4 \%)$ & 0.64 \\
Nondermatologic malignancy $^{4}(\%)$ & $6 / 68(8.9 \%)$ & 0.49 \\
\hline
\end{tabular}

${ }^{1}$ Presence or absence of clinical risk factors was determined coincident with or prior to diagnosis of skin wound. They were assessed by text review of charts for physician documentation and query of specific tests such as echocardiogram. Where presence or absence of risk factors could not be determined the information was recorded as missing. Actual numbers of recorded data for each risk factor are presented in the table. ${ }^{2}$ Healing wounds were those that healed within 1 year of followup. Non-healing wounds were defined as wounds that required amputation or did not heal after 1 year of followup. ${ }^{3}$ Immunosuppression was defined as taking immunosuppressive medications like steroids or chemotherapy or the presence of conditions of immunocompromise such as HIV/AIDS.

${ }^{4}$ Only nondermatologic malignancies co-incident with skin wound were ascertained.

*indicates statistical significance at $\alpha=0.05$.

TABLE 3: Laboratory biomarkers ${ }^{1}$ in patients with healing and non-healing wounds.

\begin{tabular}{lccc}
\hline & Healing wound & Non-healing wound & $P$ value \\
\hline Albumin $^{2}(\mathrm{~g} / \mathrm{dL})$ & $3.4 \pm 0.4$ & $2.7 \pm 0.8$ & $<0.01^{*}$ \\
$\mathrm{Hb}^{3}(\mathrm{~g} / \mathrm{dL})$ & $12.2 \pm 1.6$ & $11.3 \pm 2.2$ & $0.01^{*}$ \\
$\mathrm{WBC}^{4}\left(\mathrm{cells} / \mathrm{mm}^{3}\right)$ & $8.2 \pm 3.0$ & $10.1 \pm 3.4$ & $<0.01^{*}$ \\
Random glucose $^{5}(\mathrm{mg} / \mathrm{dL})$ & $148 \pm 59$ & $167 \pm 56$ & 0.13 \\
HbAlc $\left.^{6} \%\right)$ & $7.2 \pm 1.4$ & $8.1 \pm 2.2$ & 0.17 \\
\hline
\end{tabular}

${ }^{1}$ Laboratory measurements closest to diagnosis of ulcer but no more than 3 months prior to diagnosis were recorded.

${ }^{2}$ Based on $n=88 ;{ }^{3}$ Based on $n=121 ;{ }^{4}$ Based on $n=121 ;{ }^{5}$ Based on $n=89 ;{ }^{6}$ Based on $n=35$.

of wound also were not significantly associated with poor wound healing. Nearly all the skin ulcers studied were located on the lower extremities.

As shown in Table 2, presence of the following comorbidities at the time of ulcer diagnosis was significantly associated with the outcome: diabetes mellitus (OR 6.38), peripheral neuropathy (OR 4.17), renal insufficiency (OR 2.54), and peripheral arterial disease (OR 2.31). While patients with venous stasis ulcers tended to be more likely to have healing wounds, this relationship was not statistically significant at $\alpha=0.05$. Congestive heart failure, immunosuppression, and concurrent nondermatologic malignancy were not significantly associated with non-healing-wound outcomes.

Clinical lab data was obtained for a limited subset of the patients as detailed in Table 3. Albumin levels were significantly associated with the outcome such that patients with non-healing wounds had a mean albumin level of 2.7 compared to a mean albumin level of 3.4 for patients with healing wounds $(P<0.01)$. Hemoglobin levels were also significantly lower among patients with non-healing wounds $(P=0.01)$. WBC counts were significantly higher among patients with non-healing wounds $(P<0.01)$. Random glucose $(P=0.13)$ and HbAlc levels $(P=0.17)$ were not significantly different according to wound outcome.

Table 4 details the therapeutic interventions administered to patients with healing and non-healing wounds. Only the administration of systemic antibiotics and need for wound debridement were associated with poor woundhealing outcome. Clinical chart review of patients requiring systemic antibiotics confirmed that all these patients had developed clinical signs of secondary wound infection according to physician documentation with the exception of 2 cases. The need for surgical wound debridement likely reflected severe wounds that were at higher risk of poor healing.

Table 5 reports the independent association of clinical predictors and lab biomarkers with the outcome using multivariate logistic regression. Only the co-existing presence of diabetes (OR 5.87, 95\% CI 1.36-25.3), albumin levels (OR 0.20 per unit, 95\% CI $0.07-0.60$ ) and need for systemic antibiotics (OR 3.88, 95\% CI 1.06-14.2) remained independently predictive of the outcome after multivariate adjustment.

\section{Conclusion}

To our knowledge this is the first large study to quantify the independent impact of multiple clinical risk factors and lab biomarkers on wound outcomes in chronic skin ulcers.

Based on our preliminary analysis of 135 patients, we found that a high number of patients with chronic skin ulcers $(49.7 \%)$ seen at a tertiary care center suffered extremely poor wound-healing outcomes (amputations or non-healing wound after 1 year of followup). $36.3 \%$ of these chronic 
TABLE 4: Therapeutic interventions in patients with healing and non-healing wounds.

\begin{tabular}{lccc}
\hline & Healing wound $(n=68)$ & Nonhealing wound $(n=67)$ & $P$ value \\
\hline Topical treatments (\%) & $66 / 68(97.1 \%)$ & $63 / 67(94.0 \%)$ & 0.44 \\
Systemic antibiotics (\%) & $38 / 68(55.9 \%)$ & $55 / 67(82.1 \%)$ & $0.001^{*}$ \\
Wound debridement (\%) & $15 / 68(25.9 \%)$ & $29 / 67(43.3 \%)$ & $0.008^{*}$ \\
Peripheral revascularization (\%) & $16 / 68(23.5 \%)$ & $18 / 67(26.9 \%)$ & 0.65 \\
Compression stockings (\%) & $4 / 68(5.9 \%)$ & $6 / 67(8.9 \%)$ & 0.53 \\
Venous ablation or stripping (\%) & $5 / 68(7.4 \%)$ & $0 / 67(0 \%)$ & 0.058 \\
Skin graft (\%) & $4 / 68(5.9 \%)$ & $6 / 67(8.9 \%)$ & 0.53 \\
Wound vacuum (\%) & $4 / 68(5.9 \%)$ & $3 / 67(4.5 \%)$ & 0.99 \\
Hyperbaric oxygen (\%) & $1 / 68(1.4 \%)$ & $1 / 67(1.5 \%)$ & 0.99 \\
\hline
\end{tabular}

${ }^{1}$ Healing wounds were those that healed within 1 year of followup. Non-healing wounds were defined as wounds that required amputation or did not heal after 1 year of followup. ${ }^{2}$ Topical treatments included but were not limited to wet-dry dressings, papain ointment, topical antibiotics, silvadene ointment, whirlpool treatment, accuzyme application, and Dakin's soaks. ${ }^{3}$ Systemic antibiotics refer to antibiotics administered orally or intravenously. ${ }^{4}$ Wound debridement refers to surgical debridement or debridement in physician's office. ${ }^{5}$ Peripheral revascularization refers to angioplasty or bypass graft of peripheral arteries. ${ }^{6}$ Skin graft referred to human skin autograft or synthetic skin graft/Dermagraft. ${ }^{7}$ Hyperbaric oxygen refers to treatment in hyperbaric oxygen chamber.

*indicates statistical significance at $\alpha=0.05$.

TABLE 5: Adjusted and unadjusted odds of having a poor wound-healing outcome ${ }^{1}(N=135)$.

\begin{tabular}{|c|c|c|c|c|}
\hline \multirow{2}{*}{ Predictors } & \multicolumn{2}{|c|}{ Unadjusted } & \multicolumn{2}{|c|}{ Adjusted } \\
\hline & $\mathrm{OR}^{2}$ & $95 \% \mathrm{CI}^{3}$ & $\mathrm{OR}^{2}$ & $95 \% \mathrm{CI}^{3}$ \\
\hline Diabetes mellitus & $6.38^{*}$ & $2.89-14.1$ & $5.87^{*}$ & $1.36-25.3$ \\
\hline Peripheral neuropathy & $4.17^{*}$ & $1.99-8.76$ & 0.97 & $0.24-3.91$ \\
\hline Renal insufficiency & $2.54^{*}$ & $1.26-5.15$ & 1.32 & $0.42-4.1$ \\
\hline Need for systemic antibiotics ${ }^{4}$ & $3.62^{*}$ & $1.65-7.95$ & $3.88^{*}$ & $1.06-14.2$ \\
\hline Peripheral arterial disease & $2.31^{*}$ & $1.13-4.72$ & 1.43 & $0.45-4.52$ \\
\hline Albumin (per unit) & $0.21^{*}$ & $0.09-0.48$ & $0.20^{*}$ & $0.07-0.60$ \\
\hline Hemoglobin (per unit) & 0.79 & $0.65-0.95$ & 1.05 & $0.73-1.4$ \\
\hline
\end{tabular}

${ }^{1}$ Poor wound-healing outcome was defined as wounds that required amputation or did not heal after 1 year of followup. ${ }^{2}$ OR refers to odds ratio. ${ }^{3} 95 \%$ CI refers to $95 \%$ confidence interval. ${ }^{4}$ All cases of systemic antibiotic administration were reviewed and found to reflect secondary infection of skin wound with the exception of 2 cases which were excluded from this analysis.

*indicates statistical significance at $\alpha=0.05$.

wounds resulted in amputations. Among patients who had ulcers that ultimately healed within 1 year, median time to healing was 2 months with an interquartile range of 1-5 months. Demographic and behavioral factors such as age, sex, and current smoking status did not vary significantly for healing versus non-healing wounds. In addition, clinical features of the wound such as wound size and preexisting wound duration also did not significantly predict the outcome.

Of all the known clinical co-morbidities for poor wound outcomes such as diabetes, peripheral neuropathy, renal insufficiency, and peripheral arterial disease, the strongest association for poor outcome was diabetes. This was confirmed on multivariate adjustment indicating that diabetes confers additional risk for poor wound healing independent of peripheral neuropathy, macrovascular disease, or renal disease. This suggests that microvascular disease is very important and may indeed be the most critical factor in the pathogenesis of poor wound healing among diabetics, although it is difficult to draw causal conclusions based on this observational data. If validated by other clinical studies, this finding would indicate the need for further research to develop therapies targeted to diabetic microvascular disease.

Several lab biomarkers were significantly predictive of poor wound healing on the univariate analysis including hemoglobin, WBC count, and albumin levels. The association of WBC levels likely reflects underlying inflammation or infection while that of hemoglobin levels with wound healing suggests that local oxygen supply to a wound site through erythrocytes may be an important factor in wound healing [21-23]. However, these relationships did not remain significant on controlling for other factors.

Only low albumin levels remained significantly associated with poor wound healing upon multivariate adjustment. Hypoalbuminemia could be secondary to underlying malnutrition which would also cause poor wound healing [12]. Alternatively, the association of low albumin levels with wound healing may reflect a systemic inflammatory state in patients who go on to develop poor wound outcomes. Although there was insufficient data on ESR and CRP in this cohort, future work could focus on analyzing the role of systemic inflammation on wound healing using 
these markers. Additionally, future analyses with a larger sample size could also help reveal significant independent associations between hemoglobin levels or WBC levels with wound outcomes since limited sample sizes make it difficult to ascertain their role in wound healing in our study.

In addition to lab biomarkers and clinical comorbidities coexistent with skin ulcers, the secondary infection of these wounds and need for systemic antibiotics were a significant prognosticator of poor outcome. This strongly emphasizes that the prevention of wound infection is critical to avoiding poor outcome such as amputation in skin ulcers. In this context, early or perhaps even prophylactic antibiotic use among high-risk patients (for instance those with diabetes) may be warranted.

There were several limitations of the present study including its observational design and use of ICD-9 codes to designate skin ulcers. However, rigorous chart review to confirm clinical documentation of a skin ulcer by a physician was done in order to ensure that all those included in the study had actual skin ulcers that met our rigorous inclusion and exclusion criteria. Regarding the observational design, while an attempt was made to control known demographic, clinical, and lab risk factors, there may still be unmeasured confounding. Additionally, there was a high loss to followup in our data largely due to the nature of retrospective review of patient charts. While some patients were true losses to followup in that they did not return to Stanford or died, there were several for whom no followup data on their skin ulcers was noted. The large losses to followup from these sources introduce the possibility of selection bias in our results. Future work may include a sensitivity analysis to examine the impact of that loss to followup on our study results. Another problem associated with electronic chart review is a high rate of missing data on lab and other covariates. Since we limited the selection of lab values to those within a clinically meaningful time window in relation to the diagnosis of skin ulcers, our analyses on lab biomarkers of wound healing were limited by small sample sizes.

In the future, this analysis will be extended to additional patients discovered through the cohort discovery tool. This will increase the power for a prognostication model that will incorporate a wide range of both clinical and lab biomarkers. This will allow us to comprehensively risk stratify patients to identify those who would most benefit from early and aggressive wound care therapies such as hyperbaric oxygen, wound vacuum, or skin grafting [24-26].

\section{Acknowledgments}

The authors would like to thank Dr. Gomathi Krishnan for all her help retrieving data from the STRIDE system and Olena Mykhaylichenko for administrative assistance.

\section{References}

[1] C. K. Sen, G. M. Gordillo, S. Roy et al., "Human skin wounds: a major and snowballing threat to public health and the economy," Wound Repair and Regeneration, vol. 17, no. 6, pp. 763-771, 2009.
[2] H. Brem, O. Stojadinovic, R. F. Diegelmann et al., "Molecular markers in patients with chronic wounds to guide surgical debridement," Molecular Medicine, vol. 13, no. 1-2, pp. 30-39, 2007.

[3] D. J. Margolis, L. Allen-Taylor, O. Hoffstad, and J. A. Berlin, "The accuracy of venous leg ulcer prognostic models in a wound care system," Wound Repair and Regeneration, vol. 12, no. 2, pp. 163-168, 2004.

[4] I. C. Valencia, A. Falabella, R. S. Kirsner, and W. H. Eaglstein, "Chronic venous insufficiency and venous leg ulceration," Journal of the American Academy of Dermatology, vol. 44, no. 3, pp. 401-424, 2001.

[5] R. E. Pecoraro, G. E. Reiber, and E. M. Burgess, "Pathways to diabetic limb amputation. Basis for prevention," Diabetes Care, vol. 13, no. 5, pp. 513-521, 1990.

[6] G. E. Reiber, E. J. Boyko, and D. G. Smith, "Lower extremity foot ulcers and amputations in diabetes," in Diabetes in America, pp. 409-428, National Institutes of Health, Bethesda, Md, USA, 2nd edition, 1995, NIH Publication No. 95-1468.

[7] Centers for Disease Control and Prevention, National Diabetes Fact Sheet: General Information and National Estimates on Diabetes in the United States, 2000, US Department of Health and Human Services, Centers for Disease Control and Prevention, Atlanta, Ga, USA, 2002.

[8] H. Yasuhara, S. Naka, H. Yanagie, and H. Nagawa, "Influence of diabetes on persistent nonhealing ischemic foot ulcer in end-stage renal disease," World Journal of Surgery, vol. 26, no. 11, pp. 1360-1364, 2002.

[9] T. Mustoe, "Understanding chronic wounds: a unifying hypothesis on their pathogenesis and implications for therapy," American Journal of Surgery, vol. 187, no. 5, pp. 65S-70S, 2004.

[10] H. J. Wallace and M. C. Stacey, "Levels of tumor necrosis factor- $\alpha$ (TNF- $\alpha)$ and soluble TNF receptors in chronic venous leg ulcers-correlations to healing status," Journal of Investigative Dermatology, vol. 110, no. 3, pp. 292-296, 1998.

[11] N. J. Trengove, H. Bielefeldt-Ohmann, and M. C. Stacey, "Mitogenic activity and cytokine levels in non-healing and healing chronic leg ulcers," Wound Repair and Regeneration, vol. 8, no. 1, pp. 13-25, 2000.

[12] C. Legendre, C. Debure, S. Meaume, C. Lok, J. L. Golmard, and P. Senet, "Impact of protein deficiency on venous ulcer healing," Journal of Vascular Surgery, vol. 48, no. 3, pp. 688693, 2008.

[13] M. S. Gohel, R. A. J. Windhaber, J. F. Tarlton, M. R. Whyman, and K. R. Poskitt, "The relationship between cytokine concentrations and wound healing in chronic venous ulceration," Journal of Vascular Surgery, vol. 48, no. 5, pp. 1272-1277, 2008.

[14] M. Weckroth, A. Vaheri, J. Lauharanta, T. Sorsa, and Y. T. Konttinen, "Matrix metalloproteinases, gelatinase and collagenase, in chronic leg ulcers," Journal of Investigative Dermatology, vol. 106, no. 5, pp. 1119-1124, 1996.

[15] M. Peschen, H. Grenz, C. Grothe, E. Schöpf, and W. Vanscheidt, "Patterns of epidermal growth factor receptor, basic fibroblast growth factor and transforming growth factor$\beta 3$ expression in skin with chronic venous insufficiency," European Journal of Dermatology, vol. 8, no. 5, pp. 334-338, 1998.

[16] A. Freeman, K. May, N. Frescos, and P. R. Wraight, "Frequency of risk factors for foot ulceration in individuals with chronic kidney disease," Internal Medicine Journal, vol. 38, no. 5, pp. 314-320, 2008.

[17] D. J. Margolis, O. Hofstad, and H. I. Feldman, "Association between renal failure and foot ulcer or lower-extremity 
amputation in patients with diabetes," Diabetes Care, vol. 31, no. 7, pp. 1331-1336, 2008.

[18] P. Balaji and J. G. Mosley, "Evaluation of vascular and metabolic deficiency in patients with large leg ulcers," Annals of the Royal College of Surgeons of England, vol. 77, no. 4, pp. 270-272, 1995.

[19] A. I. Rojas and T. J. Phillips, "Patients with chronic leg ulcers show diminished levels of vitamins A and E, carotenes, and zinc," Dermatologic Surgery, vol. 25, no. 8, pp. 601-604, 1999.

[20] M. Andrews and C. Gallagher-Allred, "The role of zinc in wound healing," Advances in Wound Care, vol. 12, no. 3, pp. 137-138, 1999.

[21] K. Jonsson, J. A. Jensen, W. H. Goodson et al., "Tissue oxygenation, anemia, and perfusion in relation to wound healing in surgical patients," Annals of Surgery, vol. 214, no. 5, pp. 605-613, 1991.

[22] M. Thomas, C. Tsalamandris, R. MacIsaac, and G. Jerums, "Anaemia in diabetes: an emerging complication of microvascular disease," Current Diabetes Reviews, vol. 1, no. 1, pp. 107126, 2005.

[23] T. K. Hunt, P. Twomey, B. Zederfeldt, and J. E. Dunphy, "Respiratory gas tensions and $\mathrm{pH}$ in healing wounds," The American Journal of Surgery, vol. 114, no. 2, pp. 302-307, 1967.

[24] A. Abidia, G. Laden, G. Kuhan et al., "The role of hyperbaric oxygen therapy in ischaemic diabetic lower extremity ulcers: a double-blind randomized-controlled trial," European Journal of Vascular and Endovascular Surgery, vol. 25, no. 6, pp. 513$518,2003$.

[25] M. J. Skyhar, A. R. Hargens, and M. B. Strauss, "Hyperbaric oxygen reduces edema and necrosis of skeletal muscle in compartment syndromes associated with hemorrhagic hypotension," Journal of Bone and Joint Surgery, vol. 68, no. 8, pp. 1218-1224, 1986.

[26] J. A. Niezgoda, P. Cianci, B. W. Folden, R. L. Ortega, J. Benjamin Slade, and A. B. Storrow, "The effect of hyperbaric oxygen therapy on a burn wound model in human volunteers," Plastic and Reconstructive Surgery, vol. 99, no. 6, pp. 16201625, 1997. 


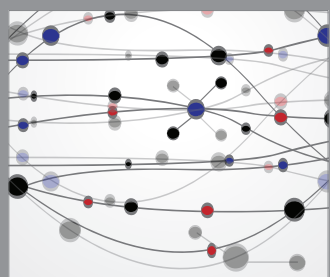

The Scientific World Journal
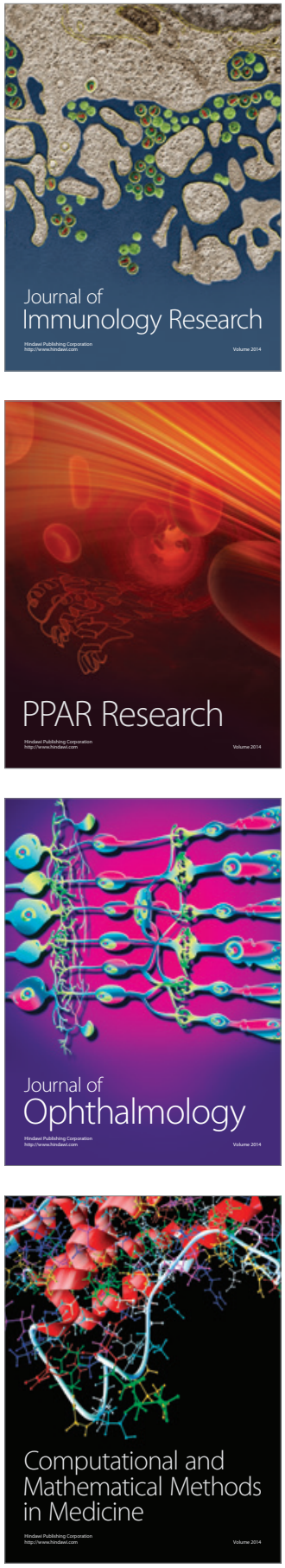

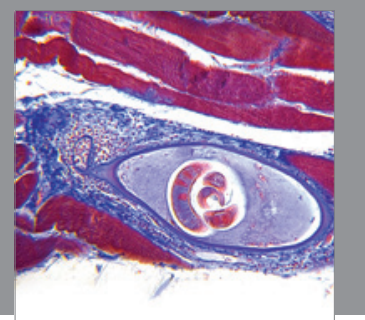

Gastroenterology

Research and Practice
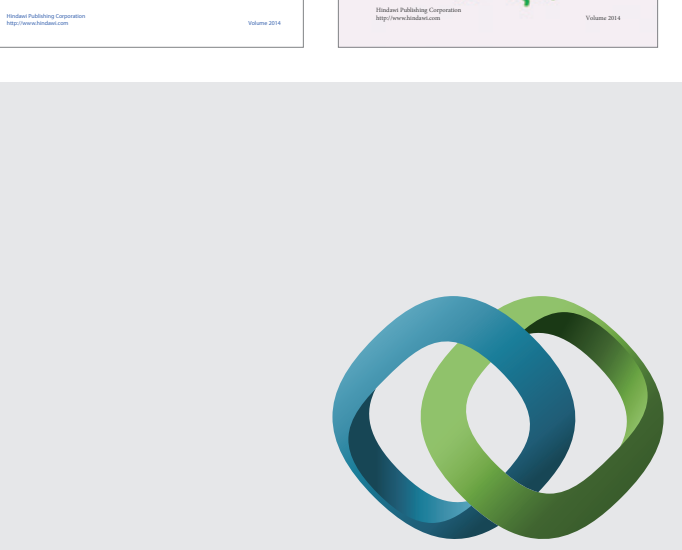

\section{Hindawi}

Submit your manuscripts at

http://www.hindawi.com
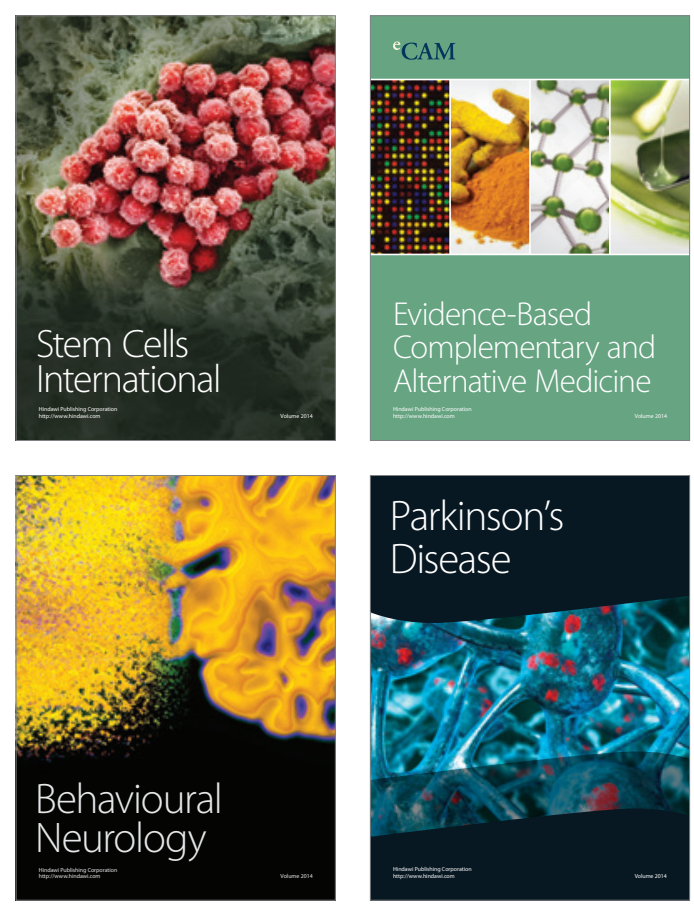

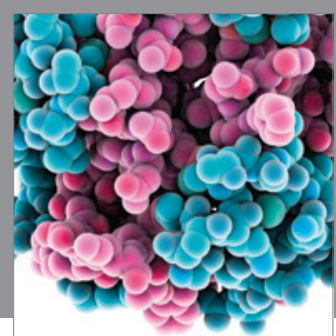

Journal of
Diabetes Research

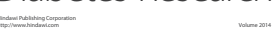

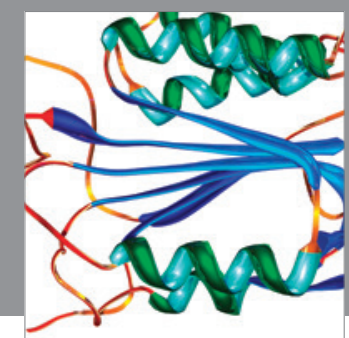

Disease Markers
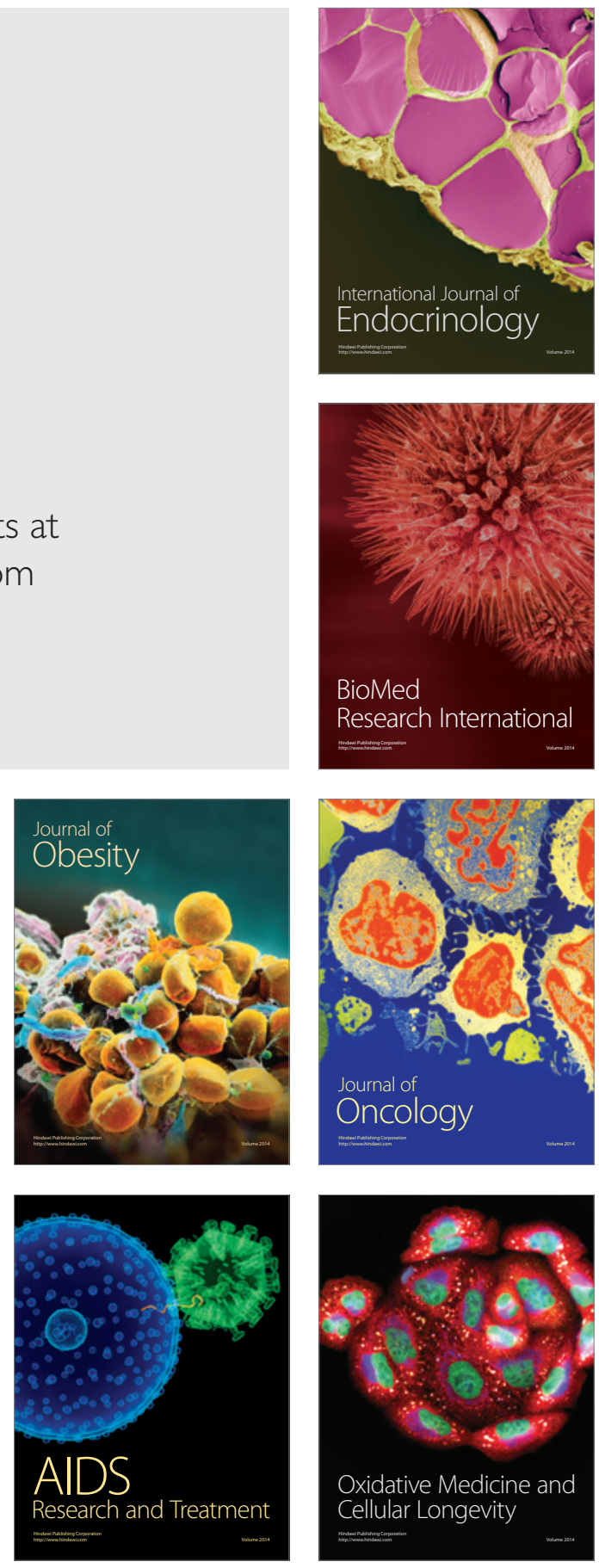